\title{
Mathematical modelling of anisotropy in fibrous connective tissue
}

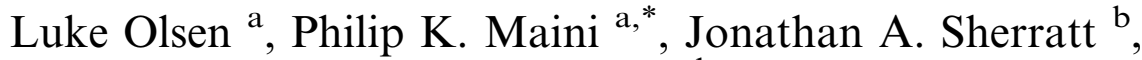 \\ John Dallon ${ }^{\mathrm{b}}$
}

a Centre for Mathematical Biology, Mathematical Institute, 24-29 St. Giles, Oxford OX1 3LB, UK

${ }^{\mathrm{b}}$ Department of Mathematics, Heriot-Watt University, Edinburgh EH14 4AS, UK

Received 7 May 1998; received in revised form 28 December 1998; accepted 28 December 1998

\begin{abstract}
We present two modelling frameworks for studying dynamic anistropy in connective tissue, motivated by the problem of fibre alignment in wound healing. The first model is a system of partial differential equations operating on a macroscopic scale. We show that a model consisting of a single extracellular matrix material aligned by fibroblasts via flux and stress exhibits behaviour that is incompatible with experimental observations. We extend the model to two matrix types and show that the results of this extended model are robust and consistent with experiment. The second model represents cells as discrete objects in a continuum of ECM. We show that this model predicts patterns of alignment on macroscopic length scales that are lost in a continuum model of the cell population. (c) 1999 Elsevier Science Inc. All rights reserved.
\end{abstract}

Keywords: Dynamic anisotropy; Matrix alignment; Wound healing; Scar tissue

\section{Introduction and biological background}

The term 'fibrous connective tissue' denotes a wide class of supporting tissues within and between organs. Common to this wide variety of tissues are fibrous extracellular matrix (ECM) and fibroblast cells. The interaction

\footnotetext{
${ }^{*}$ Corresponding author. Tel.: +44-1865 273 553; fax: +44-1865 273 583; e-mail: maini@maths. ox.ac.uk
} 
between fibroblasts and ECM plays a crucial role in a number of important biological and medical phenomena, and several mathematical models have been proposed to address various aspects of these processes. The potential for tissue mechanics to generate spatial patterns has been studied in detail by Murray, Oster and coworkers [1,2], with particular applications to embryology. The development of solid tumours within connective tissue has also been modelled extensively, including studies of angiogenesis [3], nodularity [4] and invasion $[5,6]$. There has also been extensive modelling of cell behaviour within the ECM in vitro, in particular in fibroblast-populated collagen lattices [7-9]. The vast majority of this previous modelling work assumes the ECM to be isotropic, neglecting the fact that the degree of alignment varies, with the degree of anisotropy being an important variable in the model. We are aware of only one study, other than our own recent work, in which 'dynamic anisotropy' of the ECM has been included, namely a paper by Barocas and Tranquillo [10], which we discuss later. The simpler case of tissues with a fixed degree of anisotropy has of course been studied quite extensively, in particular in models of the heart $[11,12]$.

Dynamic anisotropy is an important ingredient in a wide range of other biological systems, and has been the subject of extensive mathematical modelling in recent years. Three systems have been the focus of particular attention: the intracellular actin network, fibroblast colonies in vitro, and ecological swarming. Actin filaments are the main structural protein in cells, and exhibit preferential orientation when the cell is subject to either chemical or mechanical stimuli. This system was first modelled using a generic spatiotemporal representation [13], and subsequently in more detail but neglecting spatial variation [14,15]. In fibroblast colonies in vitro, cells re-orient as a result of direct cell-cell contacts, generating a complex range of alignment patterns, on which a large amount of detailed data is available. This has been studied extensively by Edelstein-Keshet and coworkers [16,17] using integro-differential equations. Alignment also occurs in ecological swarming of both macro-organisms and bacteria; here cellular automata are the most prevelant modelling tool $[18,19]$. Cook [20] and Grünbaum [21] have recently proposed frameworks for reducing integro-differential equations for alignment phenomena to reaction-diffusion-advection equations, which are widely applicable within both ecological and fibroblast culture contexts.

The alignment process in connective tissue differs significantly from that in these systems because of the dynamic interaction between cells and their surrounding ECM. Alignment mechanisms arising from this interaction can be broadly divided into two categories: 'flux-induced alignment' caused by the remodelling of matrix by fibroblasts as they move through the tissue, and 'stress-induced' alignment, resulting from mechanical forces within the wound. We have recently developed two different models for flux-induced alignment $[22,23]$; the present paper is concerned with stress-induced alignment and with 
the development of a single, coordinated model. The recent study by Barocas and Tranquillo [10] is the only other study that we are aware of concerning dynamic anisotropy in connective tissue. This paper does include force balance within the tissue, but the alignment they consider is due entirely to local displacements, rather than being a direct response to the stress field. The approach of Barocas and Tranquillo is in fact very different from ours, focussing on the biphasic nature of collagen gels, which consist of a fibrous network and interstitial fluid. In contrast, we neglect any role of the interstitium, and focus on the cell-fibre interactions.

The ECM anisotropy is of particular interest in the context of wound healing. Mathematical modelling has been used by a number of research groups to focus on specific aspects of the healing process. These include the regulation of wound angiogenesis [24-26], mechanochemical control of wound contraction, in which the boundaries of the wound are pulled together [27-29], and the regulation of the densities of collagen I and collagen III, which appear to control fibre thickness [30]. The ECM alignment is excluded from all these models, which focus on other aspects of the repair process. However, one of the hallmarks of scar tissue is a pronounced anisotropy in the ECM, which contrasts with the fairly isotropic surrounding dermis, and is a key indicator of scar quality; here we are referring to (an)isotropy in the fibrous ECM. During the healing process, there is a gradual transition from a non-aligned blood clot to aligned scar tissue, and an understanding of this alignment process is vital for the development of anti-scarring therapies.

We begin this paper by briefly summarising our previous work in Refs. $[22,23]$ concerning flux-induced ECM alignment. We then (Section 3) develop a simple model for stress-induced alignment, and present an analysis of equilibrium states and their stability. In Section 4, we discuss the effects of a combined model, including both stress- and flux-induced alignments. The resulting simple model is inconsistent with the observed existence of partially aligned equilibria, and in Section 5 we show that this is corrected in an enlarged model in which the interaction of two different ECM types is considered. Finally, in Section 6 we discuss a more discrete model for tissue alignment, which is concerned with alignment patterns on a microscopic length scale.

\section{Modelling flux-induced ECM alignment}

The processes by which the cells and the ECM interact to determine cell orientation and fibre alignment are very complex. It is known that cells can realign the ECM by traction $[1,32]$ and that cell motility also plays a key role in fibre alignment [31,33]. In this paper, therefore, we shall consider two forms of alignment: flux-induced alignment, in which cells remodel the matrix as they move through it, and stress-induced alignment, in which cell stress reorients 
fibre direction. We have previously developed two different continuum models for the process of flux-induced alignment, and in this section we briefly summarise these models and their equilibrium states.

\subsection{A basic model for flux-induced alignment}

We begin by reviewing the alignment model developed in Ref. [22], which is formulated in terms of the densities of the ECM fibres predominantly aligned in each of two orthogonal directions, $m_{1}(\underline{x}, t)$ and $m_{2}(\underline{x}, t)$. This is an appropriate representation for geometries in which any preferential alignment will necessarily occur along the coordinate axes: important examples include the healing of linear ('slash') and circular wounds. The representation is nevertheless restrictive. For example, it fails to distinguish between the configuration in which fibres are distributed over all possible orientations, and that in which half of the fibres are oriented in one coordinate direction, and half in the other; both of these correspond to $m_{1}=m_{2}$. We discuss below an extended model which removes this restriction by treating matrix density as a continuous function of orientation as well as space and time. However, the simpler framework has the major advantage of giving a more tractable mathematical system. In particular, we will use this framework when considering stress-induced alignment in Section 3, on the grounds of mathematical simplicity.

We thus represent matrix alignment as a reversible conversion between fibres in two orthogonal directions:

$$
m_{1} \underset{R_{1}}{\stackrel{R_{2}}{\rightleftharpoons}} m_{2} \text {. }
$$

The key assumptions are those made in the forms of $R_{1}$ and $R_{2}$; in Ref. [22], these rates were taken to be proportional to the cell flux in the 1- and 2-directions, respectively (which are the $x$ - and $y$-directions, respectively); we denote these flux components by $\mathscr{F}_{1}$ and $\mathscr{F}_{2}$. In addition it was assumed that production of new ECM in each of the 1- and 2-directions occurs at a rate $g(n, m)$, dependent on the cell density $n(\underline{x}, t)$ and the total ECM density $m(\underline{x}, t) \equiv m_{1}(\underline{x}, t)+m_{2}(\underline{x}, t)$. This gives the following equations for $m_{1}, m_{2}$ and $n$ :

$$
\begin{aligned}
& \frac{\partial m_{1}}{\partial t}=k_{\mathrm{f}}\left(-\left|\mathscr{F}_{2}\right| m_{1}+\left|\mathscr{F}_{1}\right| m_{2}\right)+g(n, m), \\
& \frac{\partial m_{2}}{\partial t}=k_{\mathrm{f}}\left(\left|\mathscr{F}_{2}\right| m_{1}-\left|\mathscr{F}_{1}\right| m_{2}\right)+g(n, m), \\
& \frac{\partial n}{\partial t}+\frac{\partial \mathscr{F}_{1}}{\partial x}+\frac{\partial \mathscr{F}_{2}}{\partial y}=f(n, m),
\end{aligned}
$$

where $k_{\mathrm{f}}$ is the flux-induced alignment rate parameter and is assumed to be a positive constant. 
Here the function $f(n, m)$ denotes the rate of cell division. In Ref. [22], the cell flux terms $\mathscr{F}_{1}, \mathscr{F}_{2}$ were based on standard representations of random cell movement, directed movement up matrix gradients ('haptotaxis') and the preferential movement of cells in directions of local ECM fibre orientation ('contact guidance'), with the specific form

$$
\left(\mathscr{F}_{1}, \mathscr{F}_{2}\right)=\overbrace{\left(\begin{array}{cc}
m_{1} / m & 0 \\
0 & m_{2} / m
\end{array}\right)}^{\text {contact guidance }}[\overbrace{\chi(m) n \frac{\partial m}{\partial x}}^{\text {haptotaxis }}-\overbrace{D(m) \frac{\partial n}{\partial x}}^{c^{\text {random }}} \begin{array}{c}
\text { movement } \\
\chi
\end{array}] .
$$

The general form of the above equation would have gradient terms for haptotaxis and for random movement. However, making the simplifying assumption that the wound is one-dimensional, that is, changes occur only in the $x$-direction, reduces the gradient terms to the above. The coefficients $\chi(m)$ and $D(m)$ both tend to zero at very high values of the matrix density $m$, which will immobilize the cells; a detailed explanation of the various terms is given in Ref. [22]. For simplicity, we scale the variables so that $n=m=1$ are the equilibrium levels in normal tissue; this implies the conditions $f(n, m)=g(n, m)=0$.

In the present paper we are primarily concerned with spatially homogeneous equilibrium states and their stability. For the model $(2 \mathrm{a}-\mathrm{d})$, this is straightforward. In a spatially homogeneous state, $\mathscr{F}_{1}$ and $\mathscr{F}_{2}$ will both be zero, and thus it can be seen immediately that $n \equiv 1, m \equiv m_{1}$ and $m_{2} \equiv 1-m_{1}$ is an equilibrium for any $m_{1} \in[0,1]$. Moreover, numerical simulations suggest that these states are all stable to spatially localised perturbations. Thus any degree of alignment is possible at a spatially uniform state, for the simple reason that the absence of cell flux means that no additional alignment takes place. For example, in the context of dermal wound healing, the basic repair process involves fibroblasts moving into the wound region from surrounding tissue, depositing collagen and aligning it in their direction of motion. However, once the wound has healed, there is no longer a net flux of fibroblasts, and consequently there is no change in the degree of alignment. Thus the anisotropy in the final healed state (the scar) depends on the degree of alignment during the transient healing process; the dependence of this on model parameter values is quantified in Ref. [22] by studying the spatiotemporal structure of the solutions during healing.

\subsection{A more detailed model for flux-induced alignment}

The basic focus of this paper is to incorporate stress-induced alignment into the model framework presented above. However, before doing so we digress briefly to review a more detailed approach to modelling flux-induced alignment developed by Dallon and Sherratt [23]. This involves treating the ECM density as a continuous function of orientation, rather than considering densities in 
two orthogonal directions, and we discuss it because of the insight given into the interpretation of the simpler framework.

In Ref. [23], the model variables are $N(\theta, t)$ and $M(\theta, t)$, denoting, respectively, cell and ECM densities as a function of time $t$ and orientation $\theta$ with respect to an arbitrary reference direction. Spatial variation could be included in principle, but is omitted in the interests of mathematical simplicity; thus the model is concerned with spatially homogeneous states, and in particular, spatially homogeneous equilibria and their stability. The conservation equation for cell density $N$ has two terms, representing random reorientation, modelled by angular diffusion, and directed reorientation, representing the tendency of fibroblasts to move preferentially in the direction of ECM orientation [34]. This directed reorientation involves a non-local convolution term, since cells and ECM can interact over a range of orientations; this use of convolutions is a fundamental feature of a number of models for alignment in other biological systems [16,35]. In the ECM conservation equation, there is only one term representing the tendency of fibroblasts to reorient collagen in their direction of motion; again, this involves a convolution. These various terms again represent the process of flux-induced alignment, although in a more detailed way than the model in Section 2.1.

Details of the mathematical model and its analysis are given in Ref. [23]; here, we simply summarise the key results. The basic implication of the model is that stable equilibria have the form of one or more localised bands of ECM orientations, with no fibres aligned at intermediate orientations. In particular, the state in which the ECM density is the same at all orientations is unstable, while the state consisting of bands of matrix aligned in two orthogonal directions is stable. There is also a wide spectrum of stable anisotropic states, consisting of one or more isolated ECM orientations, with various proportions of the ECM aligned in these discrete directions. These results are consistent with those of the simpler model presented in Section 2.1, namely that any degree of anisotropy is possible as a stable state when ECM is reoriented only by cell flux. Moreover, the more detailed framework predicts that these states consist of discrete ECM orientations, rather than a continuous range of fibre alignments. This provides specific justification for the relevance of the framework in Section 2.1, in which only two discrete, orthogonal directions are considered. In the remainder of this paper, we use this simpler representation to consider the process of stress-induced alignment, which is more complex mathematically, and thus demands a simple model framework.

\section{Basic stress-induced ECM alignment model}

The ECM fibres have the potential to be reoriented by mechanical forces within the tissue, as well as by the movement of cells. In this section, we 
consider this 'stress-induced alignment' in isolation from flux-induced alignment; in later sections, we will consider the two mechanisms acting together. Modelling alignment due to mechanical stress is significantly more complex than for flux-alignment, and we restrict attention simply to the representation of anisotropy via ECM densities in two orthogonal directions, as in Section 2.1. This is partially justified by the agreement in the flux-alignment case between the results predicted by this simple framework, and those of the more detailed model described in Section 2.2; the essential advantage of the simpler framework is its mathematical tractability.

The key assumptions in our model concern the form of the conversion rates $R_{1}$ and $R_{2}$ between the different matrix alignments: these rates were introduced in Eq. (1). Since we are considering stress-induced alignment, we suppose that $R_{1}$ and $R_{2}$ are functions of the stress tensor $\underline{\underline{\sigma}}$. For simplicity, we restrict attention to geometries in which the effective principal stress axes are always parallel to the coordinate directions: this includes a number of common wound geometries, such as a linear (slash) wound and a circular wound. Such cases avoid the need to perform a local diagonalization of $\underline{\underline{\sigma}}$ at each point in space and time in order to calculate these principal axes, and mean that we can take $R_{1}$ and $R_{2}$ to be simply a function of the diagonal, bulk components of the stress tensor, $\sigma_{11}$ and $\sigma_{22}$.

This method of including anisotropy in a mechanical model was used previously in Ref. [13], in a study of actin filament alignment. Following their approach, we impose the following constraints on the dependence on $\underline{\underline{\sigma}}$ of the alignment rates, $R_{1}$ and $R_{2}$ :

- $R_{1}$ and $R_{2}$ depend only on the ratio, $\rho=\left|\sigma_{11} / \sigma_{22}\right|$, of the magnitudes of the principal stress components;

- $R_{1}=k_{\mathrm{s}} R(\rho)$ and $R_{2}=k_{\mathrm{s}} R(1 / \rho)$, by symmetry, for some appropriate function $R$ and (constant) stress-induced alignment rate parameter $k_{\mathrm{s}}>0$;

- $R$ is an increasing function of $\rho$, with $R(0)=0$ and $R$ is bounded as $\rho \rightarrow \infty$; without loss of generality, we set this upper bound to unity.

Again following Ref. [13], we take the specific form $R(\rho)=\rho^{p} /\left(1+\rho^{p}\right)$. Here, the parameter $p>0$ is a measure of the sensitivity of the ECM alignment response to variations in the stress field.

Thus the conservation equations for $m_{1}$ and $m_{2}$ are of the form

$$
\begin{aligned}
& \frac{\partial m_{1}}{\partial t}=k_{\mathrm{s}}\left(\frac{\rho^{p} m_{2}-m_{1}}{\rho^{p}+1}\right)+g(n, m), \\
& \frac{\partial m_{2}}{\partial t}=k_{\mathrm{s}}\left(\frac{m_{1}-\rho^{p} m_{2}}{\rho^{p}+1}\right)+g(n, m),
\end{aligned}
$$

where $g(n, m)$ again represents cellular production of new ECM. We begin by considering only spatially homogeneous equilibria. In this case there will be no elastic contribution to stress, which will be due entirely to cell traction. This 
results from the pull of cells on local ECM, and its components in the 1- and 2directions will differ only in proportion to the amount of ECM aligned in these directions. Thus $\rho \equiv \sigma_{11} / \sigma_{22}=m_{1} / m_{2}$.

Again we rescale so that $n=m=1$ is the equilibrium level in normal tissue: as in the flux-aligned case, the key issue is the possible values of $m_{1}$ and $m_{2}=$ $1-m_{1}$ at equlibrium. We write $\phi=m_{1}$, so that $m_{2}=1-\phi$, giving

$$
\frac{1}{k_{\mathrm{s}}} \frac{\mathrm{d} \phi}{\mathrm{d} t}=F(\phi) \equiv \frac{\phi^{p}(1-\phi)-(1-\phi)^{p} \phi}{\phi^{p}+(1-\phi)^{p}} .
$$

Recall that $p(>0)$ is the alignment response sensitivity parameter. Equilibria occur when $F(\phi)=0$, namely for $\phi=0,1 / 2$ and $1 ; \phi=0$ and 1 correspond, respectively to matrix aligned entirely in the 1- and 2-directions, while $\phi=1 / 2$ corresponds to isotropic matrix $\left(m_{1}=m_{2}\right)$. Stability of these equilibria is easily calculated from Eq. (4) by considering the qualitative behaviour of $F$, as shown in Fig. 1. There are two scenarios, depending on the value of $p: 0$

$0<p<1: \quad \phi=0,1$ are unstable, $\phi=1 / 2$ is globally stable (subject to the constraint $0<\phi<1$ ).

$p>1$ : $\quad \phi=0,1$ are locally stable with basins of attraction $[0,1 / 2)$ and $(1 / 2,1]$ respectively; $\phi=1 / 2$ is unstable.

We thus conclude that the fully isotropic configuration will occur if the sensitivity parameter $p$ is less than the critical (bifurcation) value $p=1$, while the fully anisotropic configuration will develop if $p$ is larger than unity.

These results are similar to those of Ref. [13] for spontaneous auto-alignment of intracellular actin filaments, and are intuitively plausible: if the alignment response is sufficiently sensitive to changes in the stress field $(p>1)$ then we expect that the positive feedback that exists between stress anisotropy and matrix alignment will result in maximal alignment. However if the align-
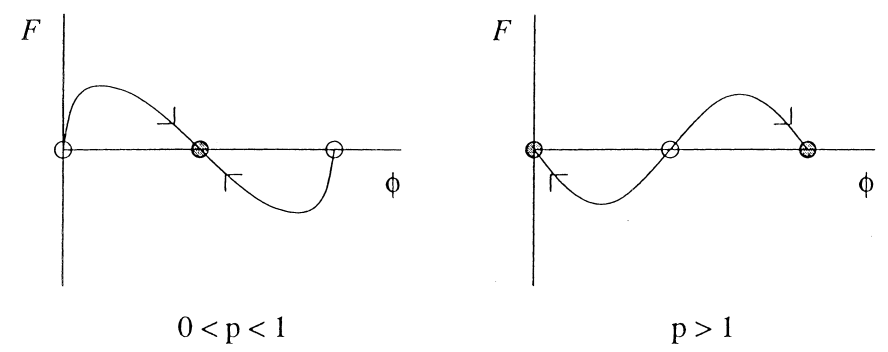

Fig. 1. The qualitative form of the function $F(\phi)$, defined by Eq. (4), for the two cases $0<p<1$ and $p>1$. The circles mark the equilibria $\phi=0,1 / 2$ and 1 , and arrows indicate the dynamics towards the locally stable equilibria (filled circles). When the alignment response is fairly insensitive to changes in the stress field $(p<1)$, the isotropic configuration $(\phi=1 / 2)$ is stable, but when the alignment response is stronger $(p>1)$, fully aligned states $(\phi=0,1)$ become stable. 
ment response is sufficiently insensitive, then the generation of stress in the dominant direction of alignment may be insufficient to overcome the intrinsic equilibrating mechanism whereby $m_{1}$ is converted into $m_{2}$ at a rate proportional to $m_{1}$, and vice versa - see Eqs. (3a) and (3b).

The isotropic architecture of normal skin corresponds to $\phi=1 / 2$ and is clearly stable to small perturbations. Thus we require $0<p<1$ in order that the model be realistic. However, a number of other tissues are highly anisotropic, in particular the scar tissue that results from dermal wound healing. This represents a stable, partially aligned state, corresponding to $1 / 2<\phi<1$, which is clearly inconsistent with the above model. In the remainder of this paper, we discuss various extensions of the model, with the aim of resolving this discrepancy. In Section 4, we consider the combined action of flux- and stress-induced alignment; this combination of the models in the current section and Section 2.1 is more realistic than either model considered in isolation, but in fact does not resolve the discrepancy. In Section 5, we study a further extension of the model, in which there are two types of interacting ECM, and show that this extension can give stable, partially aligned equilibria.

\section{Combining flux- and stress-induced alignment}

A natural extension of the models we have presented is to consider the effects of flux- and stress-induced alignment acting together. In fact the steady state structure in this case is determined only by the stress component of the alignment. This is for the simple reason that the cell flux is necessarily zero at a spatially homogeneous equilibrium. Of course, the inclusion of flux-induced alignment does alter the approach of the system towards equilibrium. To illustrate this, we digress from the consideration of homogeneous equilibria, and consider the way in which the two alignment mechanisms interact during numerical simulations of the wound healing process.

For simplicity, we restrict attention to the case of one space dimension, corresponding to a linear ('slash') wound. In this case the equations governing ECM orientation, incorporating both stress- and flux-alignment, have the form

$$
\begin{aligned}
& \frac{\partial m_{1}}{\partial t}+\overbrace{\frac{\partial}{\partial x}\left(m_{1} \frac{\partial u}{\partial t}\right)}^{\text {Convection }}=\overbrace{k_{s}\left(\frac{\sigma_{11}^{p} m_{2}-\sigma_{22}^{p} m_{1}}{\left.\sigma_{11}^{p}+\sigma_{22}^{p}\right)}\right.}^{\text {alitress }}+\overbrace{k_{f}\left|\mathscr{\mathscr { F }}_{1}\right| m_{2}}^{\text {alignent }} \begin{array}{r}
\text { Flux } \\
\begin{array}{l}
\text { ECM } \\
\text { prod }
\end{array}
\end{array} \\
& \frac{\partial m_{2}}{\partial t}+\frac{\partial}{\partial x}\left(m_{2} \frac{\partial u}{\partial t}\right)=k_{s}\left(\frac{\sigma_{22}^{p} m_{1}-\sigma_{11}^{p} m_{2}}{\sigma_{11}^{p}+\sigma_{22}^{p}}\right)-k_{f}\left|\mathscr{F}_{1}\right| m_{2}+g(n, m)
\end{aligned}
$$


where the principal stress components are

$$
\begin{aligned}
\sigma_{11} & =\overbrace{\left(E_{1}+E_{2}\right) \frac{\partial u}{\partial x}}^{\text {Elastic stress }}+\overbrace{\left(\mu_{1}+\mu_{2}\right) \frac{\partial^{2} u}{\partial x \partial t}}^{\text {Viscous stress }}+\overbrace{\tau(n, m) \frac{m_{1}}{m}}^{\text {Traction stress }} \\
\sigma_{22} & =E_{2} \frac{\partial u}{\partial x}+\mu_{2} \frac{\partial^{2} u}{\partial x \partial t}+\tau(n, m) \frac{m_{2}}{m}
\end{aligned}
$$

and the cell flux is given by

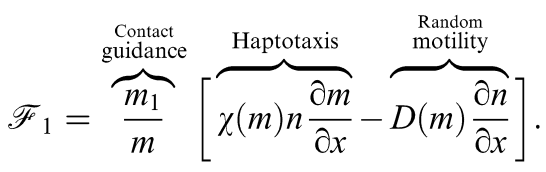

Here, $u$ is the displacement of a material point originally at a position $x$, $E_{1}=E /(1+v), E_{2}=v /(1-2 v)$, where $E$ is Young's modulus and $v$ is the Poisson ratio, $\mu_{1}$ and $\mu_{2}$ are the shear and bulk viscosities, respectively, and $\tau(n, m)$ is the tractional stress exerted by the cells. Thus, we are considering the matrix to be a linear viscoelastic material which contains cells as contractile units (see Ref. [1]). We take $\tau(n, m)$ to be proportional to cell and matrix densities when these are low, but with a decrease to zero at high densities, reflecting the phenomenon of traction inhibition [36,2]. The above is simply a combination of Eqs. (2a), (2b), (3a), (3b); the viscoelastic terms must be included in the stress tensor since we are considering the non-equilibrium situation. These equations are coupled to the conservation Eq. (2c) for cell density, which in one space dimension has the form

$$
\frac{\partial n}{\partial t}+\frac{\partial \mathscr{F}_{1}}{\partial x}=f(n, m)
$$

and to the force balance equation

$$
\frac{\partial \sigma_{11}}{\partial x}=s m u \quad \text { and } \quad \frac{\partial \sigma_{22}}{\partial x}=0 .
$$

The latter assumes that the system is operating at very low Reynolds number so that inertial terms can be ignored. Hence, the viscoelastic forces within the cellmatrix milieu are in balance with external body forces. We assume that the latter are due to elastic tethering and take the form $s m \underline{u}$, where $s$ is the modulus of elasticity, and $\underline{u}=(u, 0)$ is the displacement vector. We solve these equations on $0<x<L_{\infty}$, with $x=0$ representing the centre of a symmetric wound, and the wound edge at $x=L \ll L_{\infty}$. We use symmetry boundary conditions at $x=0$, and fix the variables at their unwounded levels at $x=L_{\infty}$, i.e. $m_{1}=m_{2}=1 / 2, n=1, u=0$. To simulate a wound, we take $n=0, m_{1}=m_{2}=$ $m_{\text {init }}$ initially inside the wound $(0<x<L)$, with unwounded levels elsewhere; 
here $m_{\text {init }}<1$ represents a low density of the ECM deposited during the initial inflammatory response to injury.

Fig. 2 illustrates the solutions of Eqs. (5a)-(5c), (6) and (7), subject to these end conditions, for a set of parameters corresponding to stress-induced alignment only $\left(k_{\mathrm{f}}=0\right)$. Cells $(n)$ move towards the wound centre as expected, depositing ECM $(m)$ in the wave-back. The cell- and ECM-mediated traction
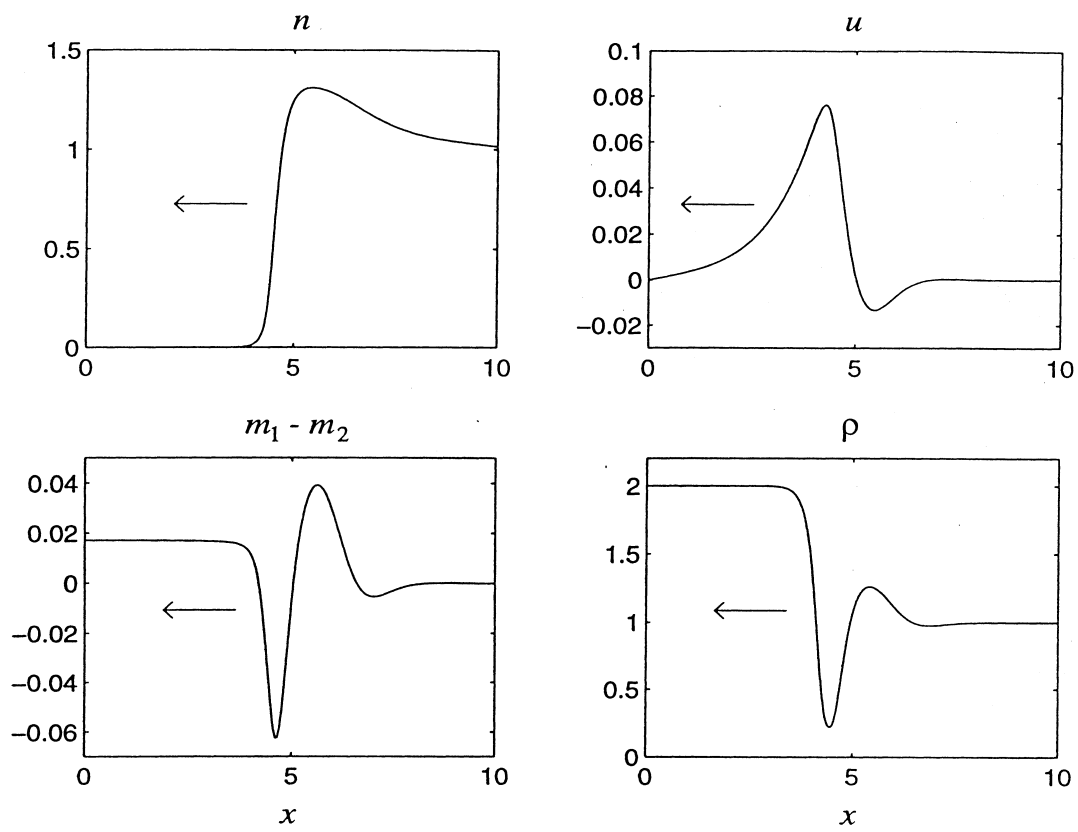

Fig. 2. Numerical simulation of dermal wound healing in the case of stress-induced alignment only, using Eqs. (5a)-(5c), (6) and (7) with $k_{\mathrm{f}}=0, k_{\mathrm{s}} \neq 0$. We plot profiles of cell density (n), tissue displacement $(u)$, the difference $m_{1}-m_{2}$ of the orthogonal ECM density components and the stress ratio $\rho=\sigma_{11} / \sigma_{22}$ at time $t=5$. The solution has the form of wave-like solutions (direction indicated by the arrows), with cells and ECM moving into the wound from its periphery. Behind this wave front, the system evolves towards the uniform, dermal steady state $n=1, m_{1}=m_{2}=\frac{1}{2}$ and $u=0$, representing normal healing with no permanent ECM alignment. The functional forms and parameter values are: $\tau(n, m)=\tau_{0} n m /\left[\left(\kappa_{\tau_{n}}^{2}+n^{2}\right)\left(\kappa_{\tau_{m}}^{2}+m^{2}\right)\right], \quad f(n, m)=n\left[-n+\alpha_{0} m /\left(\kappa_{\alpha}^{2}+m^{2}\right)\right]$, $g(n, m)=(1-m) n, D(m)=D_{0} m /\left(\kappa_{D}^{2}+m^{2}\right), \chi(m)=0, D_{0}=0.2, \kappa_{D}=0.5, \alpha_{0}=1.01, \kappa_{\alpha}=0.1$, $E_{1}=2, \quad E_{2}=2, \mu_{1}=1, \mu_{2}=1, \tau_{0}=1, \kappa_{\tau_{n}}=0.5, \quad \kappa_{\tau_{m}}=0.05, s=50, p=0.5, k_{\mathrm{s}}=10$ and $m_{\text {init }}=0.1$. The initial conditions are $n(x, 0)=(x / 10)^{h} /\left[1+(x / 10)^{h}\right], \quad m(x, 0)=m_{\text {init }}+$ $\left(1-m_{\text {init }}\right)(x / 10)^{h} /\left[1+(x / 10)^{h}\right], m_{1}(x, 0)=m_{2}(x, 0)=m(x, 0) / 2$ and $u(x, 0) \equiv 0$, with $h=50$. The boundary conditions are given by symmetry at $x=0$ and normal dermal values for the variables as $x \rightarrow \infty$, which we approximate using the infinite domain approximation $x_{\infty}=15$. Only the profiles in the region $0 \leqslant x \leqslant 10$ are shown, for clarity. The equations were solved numerically using an alternating direction semi-implicit finite difference scheme, with mesh sizes are $\Delta x=0.05$ and $\Delta t=0.001$. 
forces, together with the viscoelastic response of the ECM, cause a net stress $\left(\sigma_{11}\right)$ and thus a tissue displacement $(u)$. Ahead of the wave-front, the positive traction gradient gives rise to the positive displacement $(u>0)$ : this gradient is due to the increased traction forces generated in the wave itself compared with the relatively acellular tissue ahead of the wave. Similarly, behind the wave, traction is inhibited by the high ECM density (in particular), resulting in a negative traction gradient and hence, negative displacement $(u<0)$. The displacement is proportional to the stress gradient by virtue of the force-balance Eq. (7). Fig. 2 shows that $m_{1}>m_{2}$ if and only if $\sigma_{1}>\sigma_{2}$, or $\rho>1$. The magnitude of $m_{1}-m_{2}$ generally increases with the (stress-induced) alignment rate parameter $k_{s}$, although the effect is self-limiting due to the negative feedback property of stress-alignment which occurs whenever $p<1$, a process which accelerates with increasing $k_{\mathrm{s}}$.

In order to explain the profile of $m_{1}-m_{2}$ in Fig. 2, we consider the roles of the mechanical stress terms as detailed in Fig. 3. In the wave-front there is a
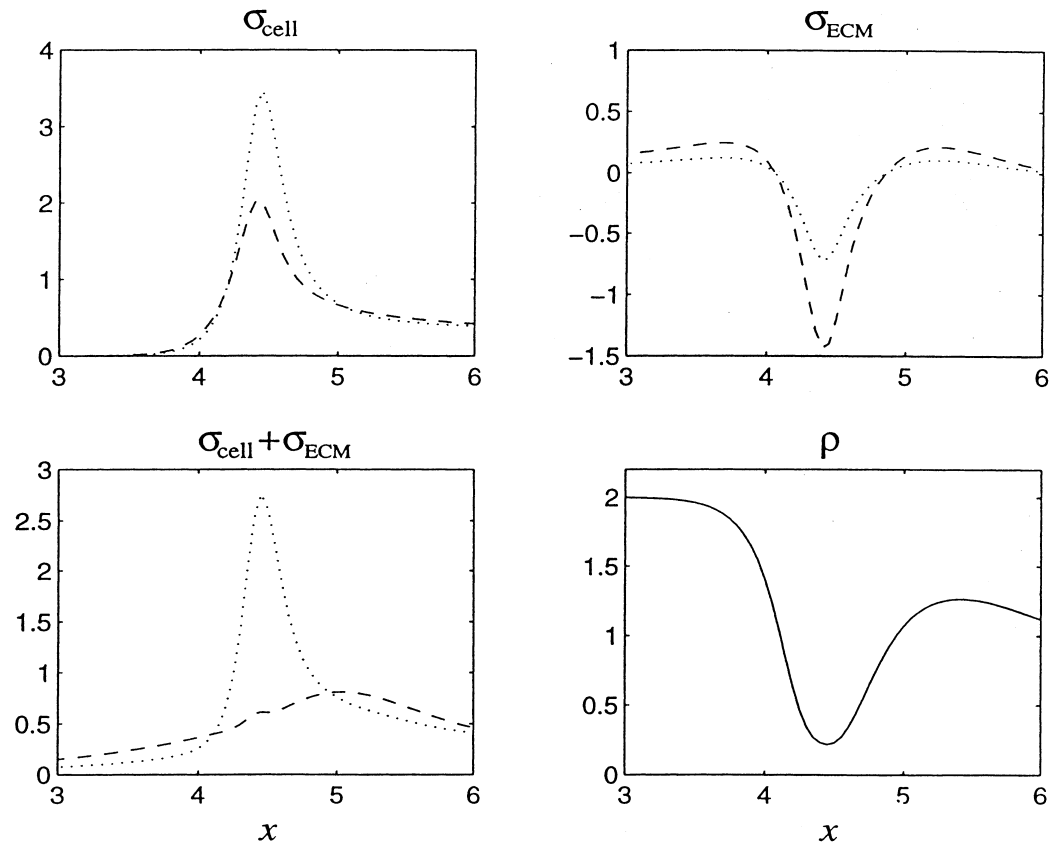

Fig. 3. Illustration of the tissue stresses implied by the numerical solutions illustrated in Fig. 2. We show profiles of the cell traction stresses (top left), ECM viscoelastic stresses (top right) and total stresses (bottom left) at time $t=5$. The $x$-axis is expanded for clarity. Components of these stress terms in the 1- and 2-directions, $\sigma_{11}$ and $\sigma_{22}$, are indicated by dashed and dotted curves, respectively. Also shown (bottom right) is the stress ratio, $\rho=\left|\sigma_{11} / \sigma_{22}\right|$, as in Fig. 2 (bottom-right). Note the sharp cell traction stress within the region $4<x<5$ which contains the position of the advancing cell front at time $t=5$ (see also Fig. 2). 
pronounced, positive cell traction profile (Fig. 3: top-left), eliciting a counteracting (negative) viscoelastic ECM response by virtue of Eq. (7). This intrinsic response is greater in the 1-direction than in the 2-direction since the dilational (bulk) stress acts in both directions whereas the shear stress acts only in the 1-direction (Fig. 3: top-right). Consequently, near the peak of the traction profile, the cell traction stress dominates the ECM stress to a greater extent in the 2-direction than in the 1-direction, causing a greater net stress in this direction (Fig. 3: bottom-left). Therefore, the ratio of absolute stresses in the 1-direction to the 2-direction is less than one in this region (Fig. 3: bottomright), thus promoting the conversion of $m_{1}$ into $m_{2}$ in this region, by Eqs. (5a) and (5b).

When flux-induced alignment is included $\left(k_{\mathrm{f}}>0\right)$, the final equilibrium level of ECM after the wave of cells has passed is still the isotropic state $m_{1}=m_{2}=1 / 2$, as expected since this remains the only stable equilibrium for $p<1$. However, the transient alignment is increased relative to the pure stressinduced alignment case, depending on the magnitudes of $k_{\mathrm{s}}$ and $k_{\mathrm{f}}$, the stressand flux-induced alignment rate parameters, respectively. For larger values of $k_{\mathrm{s}}$, the flux-induced alignment is rapidly corrected, so the spatiotemporal profiles are similar in form to those presented in Fig. 2. For lower values of $k_{\mathrm{s}}$, however, the stress-induced relaxation towards $m_{1}=m_{2}=1 / 2$ in the wake of the flux-induced effect is more gradual (illustrated in Fig. 4). If $k_{\mathrm{s}}$ is actually zero, so that only the flux-induced alignment mechanism applies, then the in-
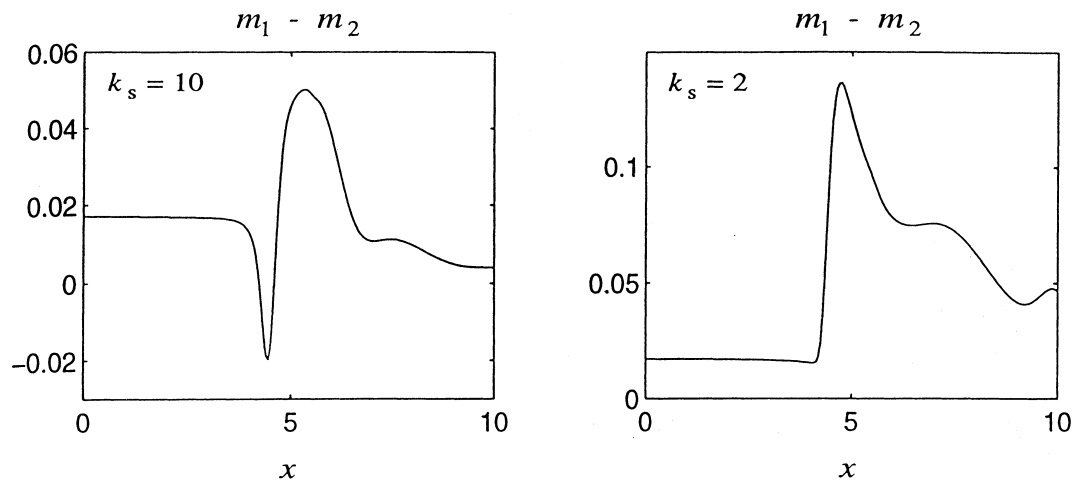

Fig. 4. Numerical simulation of dermal wound healing as in Fig. 2, but in the case of both stressinduced and flux-induced alignment, using Eqs. (5a)-(5c), (6) and (7) with both $k_{\mathrm{f}}$ and $k_{\mathrm{s}}$ non-zero. For brevity, we plot only the profiles of the difference $m_{1}-m_{2}$ of the orthogonal ECM density components. The degree of alignment $\left(m_{1}-m_{2}\right)$ within the healing wave-front is increased as a consequence of flux-induced alignment compared to the case of stress-alignment only (cf. Fig. 2). The system ultimately evolves towards the dermal steady state (cf. Fig. 2), over a longer time scale as $k_{\mathrm{s}}$ becomes smaller. The parameter values are as in Fig. 2 except for the values of $k_{\mathrm{s}}$. 
coming wave of cells leaves permanently aligned ECM behind it, with the degree of alignment dependent on details of the wave form: this dependence is explored in detail in Ref. [22].

\section{Model extension: the two ECM types}

For the combination of stress- and flux-induced alignments, only fully aligned or isotropic equilibria are stable, a property that is incompatible with the wide range of partially aligned equilibria observed in real tissues. In this section, we consider a possible resolution of this discrepancy, namely that two different ECM components may be interacting, with different alignment properties. This is particularly relevant to the proliferative phase of wound healing, during which both a collagen and a fibronectin-coated fibrin network are present in significant quantities, with fibroblasts able to realign both ECM types in vitro [37,38]. We consider first (Section 5.1) the case in which both matrix types are subject to both stress- and flux-alignment, showing that partially aligned states can then be stable. In Section 5.2 we then suppose that only one matrix type is subject to both alignment mechanisms, with the other only flux-aligned, showing that the stability of partially aligned states for two matrix types is a robust result, applying to this case also. The alternative possibility that only flux-induced alignment applies for both matrix types is trivial, with any degree of alignment possible at equilibrium.

\subsection{The two ECM types, both stress- and flux-aligned}

We denote by $m^{(1)}$ and $m^{(2)}$ the densities of the two different types of ECM; again, the subscripts ${ }_{1}$ and ${ }_{2}$ denote components in the two orthogonal directions, and suppose that the orthogonal components of $m^{(1)}$ and $m^{(2)}$ contribute linearly (additively) to the cell-derived traction stress tensor. At the dermal equilibrium where $n=1, m^{(1)}=1, m^{(2)}=1$ and $u=0$, we define $\phi=m_{1}^{(1)}$ and $\psi=m_{1}^{(2)}$. The cell traction function, $\tau$ will now be a function of three variables namely, $n, m^{(1)}$ and $m^{(2)}$. The net stress components are then given by

$$
\begin{aligned}
& \sigma_{11}=\phi \tau^{(1)}(1,1,1)+\psi \tau^{(2)}(1,1,1) \text { and } \\
& \sigma_{22}=(1-\phi) \tau^{(1)}(1,1,1)+(1-\psi) \tau^{(2)}(1,1,1)
\end{aligned}
$$

which yields the stress ratio

$$
\rho=\frac{\sigma_{11}}{\sigma_{22}}=\frac{\phi+\gamma \psi}{1-\phi+\gamma(1-\psi)}
$$

where 


$$
\gamma=\frac{\tau^{(2)}(1,1,1)}{\tau^{(1)}(1,1,1)} .
$$

We denote the alignment sensitivity parameters for $m^{(1)}$ and $m^{(2)}$ by $p$ and $q$, respectively. Then, as before, we obtain differential equations for perturbations in $\phi$ and $\psi$ about the dermal equilibrium:

$$
\begin{aligned}
& \frac{1}{k_{\mathrm{s}}^{(1)}} \frac{\mathrm{d} \phi}{\mathrm{d} t}=F(\phi, \psi) \equiv \frac{(\phi+\gamma \psi)^{p}(1-\phi)-[1-\phi+\gamma(1-\psi)]^{p} \phi}{(\phi+\gamma \psi)^{p}+[1-\phi+\gamma(1-\psi)]^{p}}, \\
& \frac{1}{k_{\mathrm{s}}^{(2)}} \frac{\mathrm{d} \psi}{\mathrm{d} t}=G(\phi, \psi) \equiv \frac{(\phi+\gamma \psi)^{q}(1-\psi)-[1-\phi+\gamma(1-\psi)]^{q} \psi}{(\phi+\gamma \psi)^{q}+[1-\phi+\gamma(1-\psi)]^{q}} .
\end{aligned}
$$

We can extend the analysis of Section 3 to this system; the details are similar and we omit them for brevity. The analysis implies that at equilibria

$$
\phi=\left[1+\left(\frac{1-\psi}{\psi}\right)^{p / q}\right]^{-1}
$$

Therefore, $0<\phi<1 / 2$ if and only if $0<\psi<1 / 2$, and $1 / 2<\phi<1$ if and only if $1 / 2<\psi<1$. Also, $\phi$ or $\psi$ are only $0,1 / 2$ or 1 when $\phi=\psi$; thus one matrix type can only be isotropic/fully aligned if the other matrix type also has this alignment. Standard local stability analysis shows that

- $(0,0)$ and $(1,1)$ are locally stable if and only if $p>1$ and $q>1$.

- $(1 / 2,1 / 2)$ is locally stable if and only if $p+\gamma q<1+\gamma$.

These results are illustrated in Fig. 5.

Using phase plane analysis we can obtain results on the global stability of these equilibria. This shows that if both the ECM types are sufficiently sensitive to variations in the local stress field, that is $p>1$ and $q>1$, then both $m^{(1)}$ and $m^{(2)}$ become fully aligned in the same direction. Conversely, if both the ECM types are relatively insensitive to the stress field, so that $p+\gamma q<1+\gamma$, then the fully isotropic, or 'random' configuration arises. This includes the possibility that either $p>1$ or $q>1$ (but not both), so that the isotropic configuration possesses a wider attracting domain in the bifurcation plane than in the analogous model with one ECM type, where this equilibrium is (globally) stable if and only if $p<1$.

The 'intermediate' situation, in which one ECM type is insensitive to the local stress field and the other is sufficiently sensitive results in a partially aligned configuration. For example, suppose that $m^{(1)}$ is insensitive to anisotropic stress and that $m^{(2)}$ is sufficiently sensitive, so that $p<1$ and $q>(1+\gamma-p) / \gamma$ : then (locally) stable equilibria $\left(m^{(1)}, m^{(2)}\right)=\left(\phi^{*}, \psi^{*}\right)$ and $\left(1-\phi^{*}, 1-\psi^{*}\right)$ occur where $0<\psi^{*}<\phi^{*}<1 / 2$, indicating that $m^{(1)}$ is aligned to a lesser extent than $m^{(2)}$. 


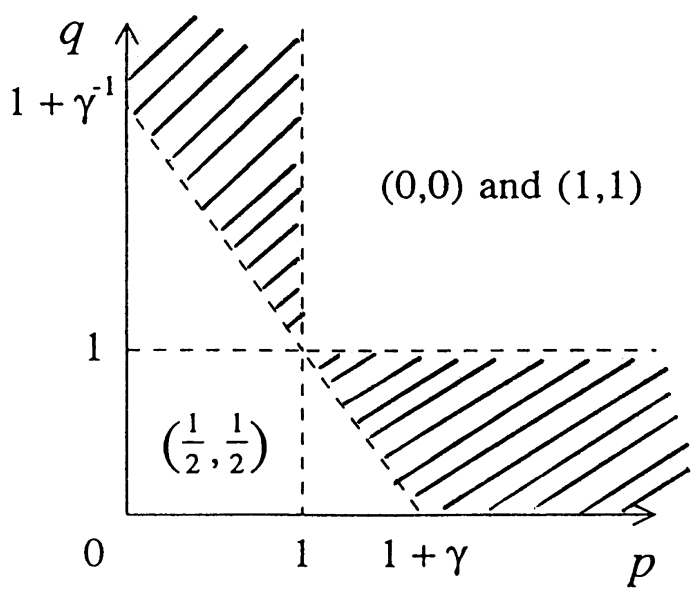

Fig. 5. An illustration of the regions in $p-q$ space in which the steady states $(\phi, \psi)=(0,0)$, $(1 / 2,1 / 2)$ or $(1,1)$ are (locally) stable. The shaded regions indicate where all three equilibria are unstable; in these regions, detailed phase plane analysis reveals the existence of at least one locally stable equilibrium in the region $0<\phi, \psi<1 / 2$, with a corresponding equilibrium in $1 / 2<\phi, \psi<1$.

\subsection{Two ECM types, one only flux-aligned}

We now consider the possibility that two matrix types interact, but with only one subject to stress-induced alignment, with the other aligned only by cell flux. The object of this extension is to consider the robustness of the potential stability of partially aligned equilibria. We suppose that the orthogonal components of $m^{(1)}$ and $m^{(2)}$ contribute linearly (additively) to the cell-derived traction stress tensor, as in the previous model. However, in this case, the components of net stress feed back only into the rate of conversion of the orthogonal components of $m^{(1)}$, with $m^{(2)}$ subject only to flux-induced alignment (previously, both of the ECM types were stress-aligned as well as flux-aligned).

We suppose that the traction stresses are given by

$$
\sigma_{i i}=\frac{m_{i}^{(1)}}{m^{(1)}} \tau^{(1)}\left(n, m^{(1)}, m^{(2)}\right)+\frac{m_{i}^{(2)}}{m^{(2)}} \tau^{(2)}\left(n, m^{(1)}, m^{(2)}\right)
$$

for $i=1,2$ with $\tau^{(1)}, \tau^{(2)} \geqslant 0$. We also assume that only $m^{(1)}$ is stress-aligned via the mechanism discussed in Section 3, that both types may be flux-aligned and that the dermal equilibrium density of each ECM type has been scaled to unity. At this equilibrium (where cell density $n \equiv 1$ and there in no net tissue displacement), the stress ratio is given by

$$
\rho=\frac{\sigma_{11}}{\sigma_{22}}=\frac{\gamma_{1}+\phi}{\gamma_{2}+1-\phi},
$$


where

$$
\gamma_{1}=\frac{\tau^{(2)}(1,1,1) m_{1}^{(2)}}{\tau^{(1)}(1,1,1)} \text { and } \gamma_{2}=\frac{\tau^{(2)}(1,1,1) m_{2}^{(2)}}{\tau^{(1)}(1,1,1)}=\frac{\tau^{(2)}(1,1,1)\left(1-m_{1}^{(2)}\right)}{\tau^{(1)}(1,1,1)}
$$

and $\phi$ is the equilibrium value of $m_{1}^{(1)}$. Since matrix of type 2 is only fluxaligned, $m_{1}^{(2)}$ can take any value (subject to $0<m_{1}^{(2)}$ ) at equilibrium. Therefore, $\gamma_{1}$ and $\gamma_{2}$ may be regarded as parameters, and we investigate the range of possible values for $\phi$ and the stability of these equilibria, as $\gamma_{1}, \gamma_{2}$ and $p$ vary.

To begin, we use the above formulae to derive the differential equation governing (temporal) perturbations in $\phi$ about the dermal equilibrium, giving

$$
\frac{1}{k_{\mathrm{s}}} \frac{\mathrm{d} \phi}{\mathrm{d} t}=F\left(\phi ; \gamma_{1}, \gamma_{2}, p\right) \equiv \frac{\left(\gamma_{1}+\phi\right)^{p}(1-\phi)-\left(\gamma_{2}+1-\phi\right)^{p} \phi}{\left(\gamma_{1}+\phi\right)^{p}+\left(\gamma_{2}+1-\phi\right)^{p}} .
$$

Note that the denominator is positive since the parameters $\gamma_{1}, \gamma_{2}$ and $p$ are nonnegative and $0 \leqslant \phi \leqslant 1$, and that the function $F(\phi)$ is continuous on this interval. Also, $F(0) \geqslant 0$ and $F(1) \leqslant 0$, with equality if and only if $\gamma_{1}=0$ and $\gamma_{2}=0$, respectively. This implies the existence of at least one locally stable equilibrium in the confined set $0<\phi<1$ for the case $\gamma_{1}, \gamma_{2}>0$, and that an odd number of equilibria exist in this interval.

Detailed investigation of equilibria requires care. In particular, the number of equilibria depends on parameter values. The boundaries in parameter space between different numbers of equilibria will correspond to repeated steady state solutions of (9), and solving $F=\mathrm{d} F / \mathrm{d} \phi=0$ gives a family of curves in the $\left(\gamma_{1}, \gamma_{2}\right)$ plane, expressed parametrically in terms of $\phi$ in the following form:

$$
\begin{aligned}
& \gamma_{1}=\Gamma_{1}(\phi ; p)=p \phi(1-\phi)\left[1+\left(\frac{1-\phi}{\phi}\right)^{1 / p}\right]-1+\phi, \\
& \gamma_{2}=\Gamma_{2}(\phi ; p)=p \phi(1-\phi)\left[1+\left(\frac{\phi}{1-\phi}\right)^{1 / p}\right]-\phi .
\end{aligned}
$$

When $p<1$, a straightforward check confirms that $\Gamma_{1}$ and $\Gamma_{2}$ are never both positive, so that there are no changes in the number of equilibria. Moreover, when $p \ll 1, F(\phi) \approx 1 / 2-\phi$, so that $\phi \approx 1 / 2$ is the unique equilibrium. Thus for any $p<1$, there is a unique equilibrium, which is globally stable. This equilibrium varies monotonically from $1 / 2$ to $\gamma_{1} /\left(\gamma_{1}+\gamma_{2}\right)=m_{1}^{(2)}$ as $p$ increases from 0 to 1 . This is as expected intuitively, since $p$ measures the sensitivity of $m^{(1)}$ alignment to anisotropy in the stress field. Thus when $p \ll 1$, changes in stress have relatively little effect. As $p$ increases, $m^{(1)}$ becomes aligned sympathetically to the alignment of $m^{(2)}$, with the alignments becoming the same at $p=1$; recall that $m^{(2)}$ is flux-aligned only, with $m^{(1)}$ subject to both alignment mechanisms. 
As $p$ increases above 1, multiple equilibria are possible, depending on the values of $\gamma_{1}$ and $\gamma_{2}$. When these parameters are sufficiently large, there is a single equilibrium, with the value of $\phi$ reflecting sympathetic alignment of $m^{(1)}$ and $m^{(2)}$. However, when $p>1$, the curve defined by (10) now lies in the positive quadrant of the $\gamma_{1}-\gamma_{2}$ plane; the form of this curve is illustrated in Fig. 6, showing in particular the cusp at $\gamma_{1}=\gamma_{2}=(p-1) / 2$. This point corresponds to $\phi=1 / 2$, and a cusp, or fold, bifurcation occurs there. For $\gamma_{1}$ and $\gamma_{2}$ lying below this critical point there are three possible equilibrium values of $\phi \in(0,1)$ : two are locally stable and their basins of attraction are separated by the third, unstable equilibrium. That the bifurcation is from one to three (rather than $>3$ ) equilibria can be shown by continuity, with reference to the special case $p \rightarrow \infty$. In this limit the bifurcation curve (10) approaches the two parallel lines $\gamma_{2}=\gamma_{1} \pm 1$, and determination of the limiting form of $F$ shows that there are three equilibria for $\gamma_{1}, \gamma_{2}$ between these lines, namely 0,1 and $\left(1-\gamma_{1}+\gamma_{2}\right) / 2$. For $\gamma_{1}, \gamma_{2}$ above/below both of these lines, $\phi=0 / 1$ is the only equilibrium. Thus as $p \rightarrow \infty$, only fully aligned states are stable; this is expected intuitively, since large $p$ corresponds to strong sensitivity to anisotropy in the stress field.

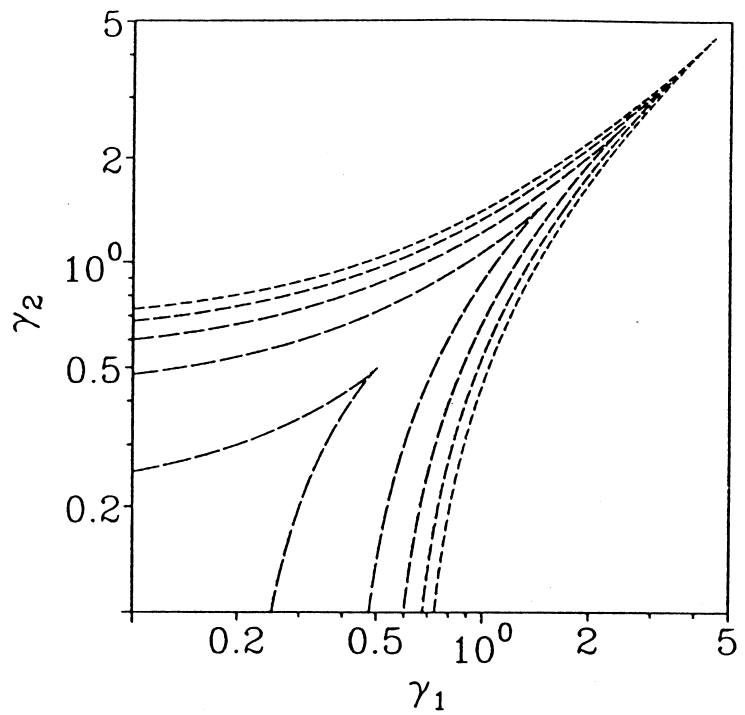

Fig. 6. Plots of the parametric curves defined (in the positive quadrant) by $\gamma_{1}(\phi ; p)$ and $\gamma_{2}(\phi ; p)$, for $p=2,4,6,8$ and 10 (increasingly broken curves), delineating the regions in which there exist one (to the top-right of the curves) and three (to the bottom-left of the curves) equilibria in the interval $0<\phi<1$. We use logarithmic axes, for clarity. The cusp bifurcations at $\gamma_{1}=\gamma_{2}=(p-1) / 2$ are apparent from these curves. 
Variant: non-linear traction coupling: We briefly consider a different scenario in which the components of the two ECM types contribute multiplicatively rather than additively (or linearly) to the traction stress. In the context of wound healing, this is partly motivated by the co-distribution of fibronectin and collagen fibres in wound tissue [39,40]. It is believed that adhesion complexes involving collinear extracellular fibronectin and collagen fibrils at cell surfaces may coordinate the intracellular and extracellular mechanical responses which result in macroscopic cell-derived traction forces $[41,42,37,43]$.

Such observations suggest the hypothesis that both collagen and fibronectin must be aligned (to some extent) in a given direction for cells to exert traction forces in that direction. In this scenario, the simplest expression for the traction stress components is a multiplicative coupling of the components of the two ECM types, given by

$$
\sigma_{i i}=\frac{m_{i}^{(1)}}{m^{(1)}} \frac{m_{i}^{(2)}}{m^{(2)}} \tau\left(n, m^{(1)}, m^{(2)}\right)
$$

for $i=1,2$ (and $\tau \geqslant 0$ ). The stress ratio, previously given by Eq. (21), now becomes

$$
\rho=\frac{\phi m_{1}^{(2)}}{(1-\phi) m_{2}^{(2)}} .
$$

Determination of steady states and their stability is straightforward; we omit the details for brevity and simply summarise the results. When $p>1$, the fully aligned equilibria $\phi=0,1$ are both stable, separated by a third unstable equilibrium. Conversely, for $p<1$, it is this intermediate state that is stable, and with a preferential alignment for $m^{(1)}$ mirroring that for $m^{(2)}$; as $p \rightarrow 0$, $\phi \rightarrow 1 / 2$, corresponding to an isotropic state.

\subsection{Conclusions}

We have shown that the interaction between cells and two types of ECM enables a partially aligned state to be a stable equilibrium in the presence of stress-induced alignment. This is true whether one or both of the matrix types is aligned by the stress field, although in the latter case, the alignment properties of the two matrix types must be significantly different. This suggests that where partial ECM anisotropy is observed, two ECM components with different alignment properties must be present and interacting. In the context of dermal wound healing, it is well known that collagen and fibronectin-coated fibrin matrices act in combination throughout the healing process; our modelling predicts that differences in the alignment properties of these matrix components may be crucial in establishing the alignment patterns that are observed in scar tissue. Even in the case of two ECM types, one feature not 
exhibited by the model is the coexistence of stable partially aligned and isotropic states. For wound healing, this is a significant omission, since aligned scar tissue coexists with isotropic surrounding dermis. The model thus suggests that this inconsistency with experimental observations reflects different alignment properties in the two tissues.

\section{Alignment at the microscopic scale}

The models considered so far have all involved continuum representations of cell and matrix density. In this section, we discuss a discrete model for alignment, whose aim is to address the possibility of alignment on a smaller scale. Although many connective tissues are spatially homogeneous and isotropic at a macroscopic level, they nevertheless have spatial structure and localised anisotropy at the microscopic level. Our objective in this section is to show that a different type of mathematical model can reveal this local structure, which is entirely masked by continuum models. We restrict attention to fluxinduced alignment; the effect of anisotropic stress at this smaller scale is an important but challenging question that we hope to consider in subsequent work. In this new model, spatial variation is a key ingredient. However, we must emphasise that the spatial scale here is much smaller than in the previous model frameworks, and that the size of domain we consider $\left(1 \mathrm{~mm}^{2}\right)$ would be averaged to a single point in the continuum models described above. For simplicity, we restrict attention to two spatial dimensions: this has the great advantage that orientations can be expressed as a single real number. In this two-dimensional domain, we represent cells as discrete objects, while ECM is treated as a continuous variable. We make the simplifying assumption that ECM density does not vary with position, and include variation only in the orientation of the matrix. Thus the model variables are the positions $\underline{P}_{i}(t)$ $(1 \leqslant i \leqslant K)$ of the $K$ cells, their directions of motion $D_{i}(t)(1 \leqslant i \leqslant K)$, and the ECM orientation $\Theta(\underline{x}, t) ; D_{i}$ and $\Theta$ are angles measured with respect to the same reference direction, and to be specific, we take this to be the $x$-coordinate direction in our two-dimensional domain. Note that we assume a single dominant orientation for the matrix at each space point; this is a reasonable assumption at this smaller spatial scale.

Within this framework, flux-induced alignment is represented very simply, as a change in $\Theta$ towards the directions $D_{i}$ of cells near that point in space. This reorientation will in fact involve a time lag, because the time taken for ECM reorientation corresponds to the cell moving a distance that is significant on the small scale we are considering. In practice, fibroblasts have significantly elongated shapes, with direction determined at the front of the cell while remodelling occurs at the rear [44]; the time lag gives a measure of this elongation. This gives an equation for $\Theta$ of the form 


$$
\frac{\partial \Theta(\underline{x}, t)}{\partial t}=\kappa \sum_{i=1}^{K} W\left(\underline{P}_{i}(t)-\underline{x}\right) \Psi\left[D_{i}\left(t-t_{\mathrm{lag}}\right)-\Theta(\underline{x}, t)\right],
$$

where $t_{\text {lag }}$ is the time lag, which we assume to be constant. Here we assume a linear superposition of cell activity. This simplifying assumption is realistic provided cell densities are relatively low; at very high cell densities, new phenomena arise via direct cell-cell interactions, which require a quite different type of model formulation [35,17]. The function $W$ is a weighting function that decreases with the distance between the cell and the space point concerned; we take $W$ to be zero at distances greater than about $10 \mu \mathrm{m}$ (about one cell length), so that only cells within this distance of a point can influence the ECM alignment there. The function $\Psi$ is linear when $D_{i} \approx \Theta$, but on a larger scale $\Psi$ is periodic, reflecting the fact that cell $i$ is aligned with the local ECM whenever $D_{i}$ and $\Theta$ differ by a multiple of $\pi$. The parameter $\kappa$ reflects the extent of cellular reorientation of matrix, and is the key parameter of the model.

Cell position changes simply because of cells moving in their current direction; we take cell speed to be a constant $S$, so that

$$
\frac{\mathrm{d} \underline{P}_{i}}{\mathrm{~d} t}=S\left(\cos D_{i}, \sin D_{i}\right) \quad(1 \leqslant i \leqslant K) .
$$

The direction of the cells will also change of course, because of their tendency to move up ECM fibres. We model this 'contact guidance' by assuming that cell direction aligns exactly with the ECM orientation; thus we take

$$
D_{i}(t)=\Theta\left(\underline{P}_{i}(t), t\right) \quad(1 \leqslant i \leqslant K) .
$$

This equation for $D_{i}$ completes the formulation of the model; further details will be presented elsewhere [45]. From Eq. (11c), it is clear that the time lag $t_{\text {lag }}$ plays an important role in the model; in particular, if $t_{\text {lag }}=0$, the cells orient the ECM towards the current cell orientation, which is simply that of the ECM, resulting in no restructuring of the matrix. At non-zero $t_{\mathrm{lag}}$, however, the alignment of the matrix is significant. A natural extension of the model would be to incorporate some persistence into the fibroblast movement, replacing Eq. (11c) with a differential equation in which $\mathrm{d} D_{i} / \mathrm{d} t$ is related to $\left[\Theta\left(P_{i}, t\right)-D_{i}\right]$; in fact we have found that such extensions do not alter the qualitative nature of the results. Further details of numerical methods for this type of model are given in Ref. [46].

We solve Eqs. (11a)-(11c) numerically on a square domain; to represent spatial homogeneity, we use periodic boundary conditions. Numerically, there is a fixed space grid for the ECM, but the cells are allowed to take any positions, rather than being confined to grid points. Eq. (11c) then requires the value of ECM orientation $\Theta$ between grid points, and we determine this using a tensor product interpolant with quartic Lagrangian interpolation in each 
direction [47]. We take the matrix to be isotropic with two orthogonal orientations initially, reflected in numerical simulations by randomly choosing the initial value of $\Theta$ at each point in the spatial grid to be one of the two directions. The cells are initially placed uniformly in the domain.

Fig. 7 illustrates a range of solutions of Eqs. (11a)-(11c), solved numerically in this way, using 196 cells in a domain of size $1 \mathrm{~mm} \times 1 \mathrm{~mm}$. We illustrate the matrix orientations by plotting a series of short curve segments with tangents whose angle is equal to the matrix alignment $\Theta$ at each point; in the language of fluid dynamics, these curve segments are streamlines. The most significant aspect of this alignment pattern is the variation with $\kappa$, which measures the extent to which the ECM is reoriented by the cells. When $\kappa$ is low, the matrix remains in a disorganised state, similar to the initial random state; this is exactly as expected, since at low $\kappa$ the cells have little effect on the matrix through which they are moving. As $\kappa$ is increased, patterns of alignment develop. The whole domain does not become aligned, however; rather, a series of small regions of roughly unidirectional alignment develop, on a length scale which increases as $\kappa$ increases approaching a value of about $250 \mu \mathrm{m}$.

\section{Discussion}

In this paper we have presented a number of ways to model cell-matrix anisotropic interactions. The main body of the paper deals with a continuumtype model which considers alignment on a macroscopic scale. In Section 6 we consider a novel discrete model on the microscopic scale. The simulations of this model show that the alignment patterns predicted by the macroscopic models discussed in previous sections of the paper can in fact mask a pattern of microscopic alignment, occuring on a scale of a few cell lengths. The occurrence of such patterns depends on the strength of the flux-induced alignment, requiring that the matrix orientation is sufficiently sensitive to cell movements. These microscopic alignment patterns will be superimposed on the macroscopic patterns described in previous sections of the paper, and may play an important role in the properties of the tissue. Important challenges raised by these results include the incorporation of stress into this microscopic model, and the extension of a model reflecting this microscopic detail to a macroscopic scale; this latter problem is straightforward in principle, but leads to an unfeasible computational problem. Furthermore, cell motion arises as a result of the mechanical interaction between cells and matrix, which is mediated through cell traction. Therefore, from a detailed mathematical description of this interaction, one should be able to recover the flux term. At the moment, such a detailed description does not exist, but its derivation would have application in many areas of mathematical biology in which cell motion is important. 

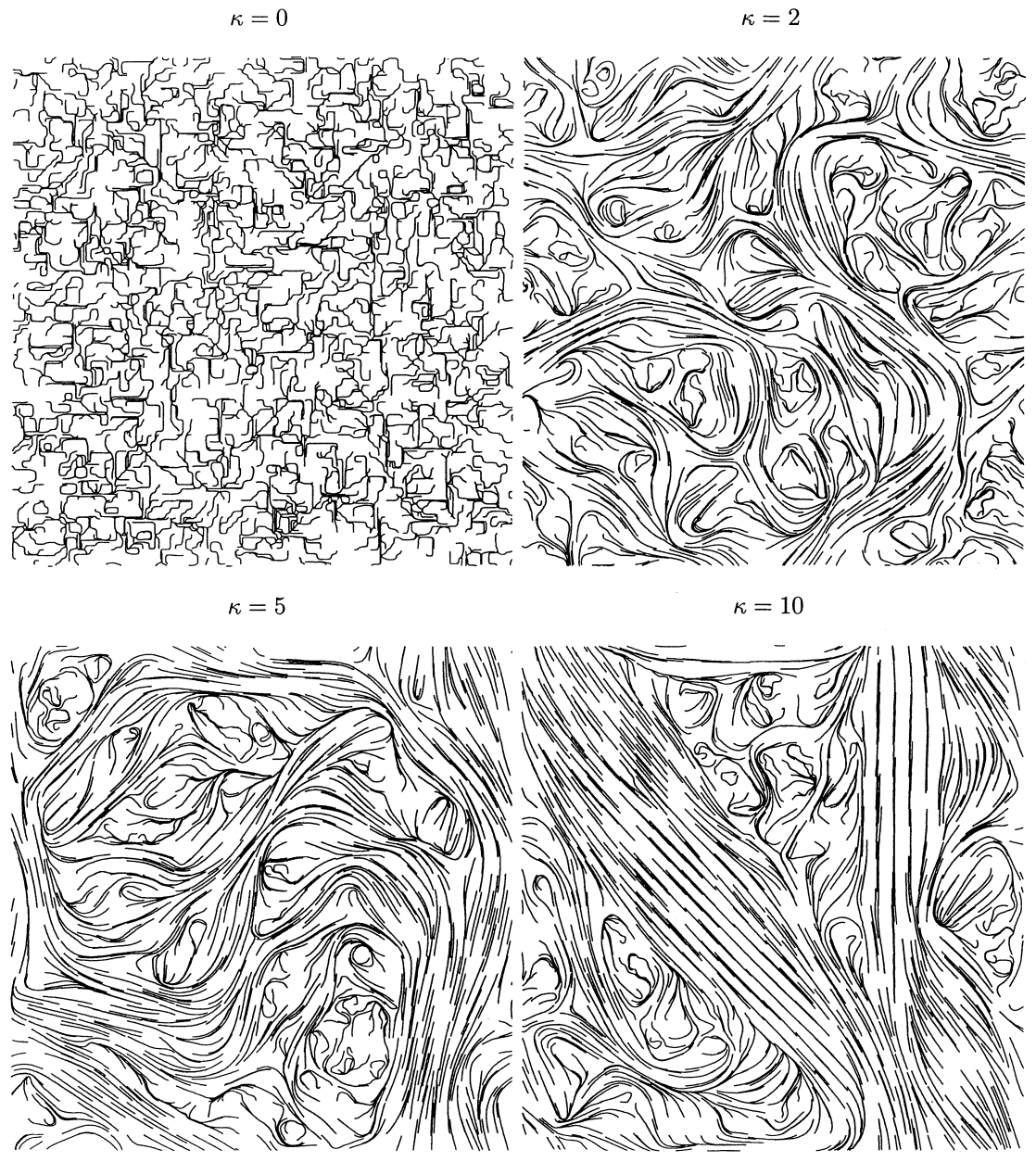

Fig. 7. The ECM orientation is shown for the discrete cell model with various values of $\kappa$. In the top left $\kappa=0$, so that the initial configuration of the ECM is preserved; in the top right $\kappa=2$, in the bottom left $\kappa=5$ and in the bottom right $\kappa=10$. As $\kappa$ increase the regions of alignment become larger. The cell speed is $40 \mu / \mathrm{h}, \Psi(\theta)=\sin \theta$ and $W\left(P_{i}-x\right)=a_{x} a_{y}$ with $a_{x}=\max \left\{\left(1-\left|p_{x}-x\right| / L\right), 0\right\}$ and $a_{y}=\max \left\{\left(1-\left|p_{y}-y\right| / L\right), 0\right\}$ where $\underline{P}_{i}=\left(p_{x}, p_{y}\right), \underline{x}=(x, y)$ and $L$ is a typical cell diameter, taken to be $10 \mu \mathrm{m}$. There are 101 grid points in each direction and the time step and $t_{\text {lag }}$ are both $0.15 \mathrm{~h}$. The figure shows solutions after a long time $(1000 \mathrm{~h})$, allowing transients to dissipate. It is important to stress that the figure does not show permanent equilibria, since the solutions continue to change dynamically as the cells move through the domain. As time progresses, effects from the periodic boundary conditions will dominate the solution; however, by changing the boundary conditions to no flux where the cells are randomly allowed to re-enter the domain when they leave, the same qualitative features of the solution appear and persist. By examining both boundary conditions, it can be seen that the structure is not introduced by the randomness in the no flux boundary conditions and gives confidence that this structure would be present for simulations with an infinite domain. If the initial conditions are altered such that the ECM orientation is random, the same features are present. 
For the models on the macroscopic scale, we have considered two different matrix components and assumed that their re-alignment depends only on cellmatrix interactions. In reality, the matrix networks will interact with each other so that a more realistic model should consider how the reorientation of one matrix component affects other matrix components.

\section{Acknowledgements}

L.O. would like to thank the EPSRC for an Earmarked Studentship in Mathematical Biology. This work was supported by grant GR/K71394 from the EPSRC, and by a grant from the London Mathematical Society (scheme 3). We thank Professor Mark Ferguson (University of Manchester) for helpful discussions.

\section{References}

[1] J.D. Murray, G.F. Oster, Cell traction models for generating pattern and form in morphogenesis, J. Math. Biol. 19 (1984) 265.

[2] J.D. Murray, P.K. Maini, R.T. Tranquillo, Mechanochemical models for generating biological pattern and form in development, Phys. Rep. 171 (1988) 59.

[3] M.E. Orme, M.A.J. Chaplain, A mathematical model of the first steps of tumour-related angiogenesis: capillary sprout formation and secondary branching, IMA J. Math. Appl. Med. Biol. 13 (1996) 73.

[4] A.J. Perumpanani, J.A. Sherratt, J. Norbury, Mathematical modelling of capsule formation and multinodularity in benign tumour growth, Nonlinearity 10 (1997) 1599.

[5] P. Tracqui, D.E. Woodward, G.C. Cruywagen, J. Cook, J.D. Murray, A mechanical model for fibroblast-driven wound healing, J. Biol. Systems 3 (1995) 1075.

[6] A.J. Perumpanani, J.A. Sherratt, J. Norbury, H.M. Byrne, Biological inferences from a mathematical model for malignantinvasion, Invasion Metastasis 16 (1996) 209.

[7] R.B. Dickinson, R.T. Tranquillo, A stochastic model for adhesion-mediated cell random motilityand haptokinesis, J. Math. Biol. 31 (1993) 563.

[8] V.H. Barocas, A.G. Moon, R.T. Tranquillo, The fibroblast-populated collagen microsphere assay of celltraction force - part 2: Measurement of the cell traction parameter, J. Biomech. Eng. 117 (1995) 161.

[9] I. Ferrenq, L. Tranqui, B. Vailhe, P.Y. Gumery, P. Tracqui, Modelling biological gel contraction by cells: Mechanocellular formulation and cell traction force quantification, Acta Biotheoret. 45 (1997) 267.

[10] V.H. Barocas, R.T. Tranquillo, An anisotropic biphasic theory of tissue-equivalent mechanics: The interplay among cell traction fibrillar network deformation fibril alignment and cell contact guidance, J. Biomech. Eng. Trans. ASME 119 (1997) 137.

[11] S. Veronese, H. Othmer, A computational study of wave propagation in a model for anisotropic cardiac ventricular tissue, Lect. Notes Comp. Sci. 919 (1995) 248.

[12] A.T. Winfree, Heart muscle as a reaction-diffusion medium: The roles of electric potential diffusion activation front curvature and anisotropy, Int. J. Bifurc. Chaos 7 (1997) 487. 
[13] J.A. Sherratt, J. Lewis, Stress-induced alignment of actin filaments and the mechanicsof cytogel, Bull. Math. Biol. 55 (1993) 637.

[14] G. Civelekoglu, L. Edelstein-Keshet, Modelling the dynamics of F-actin in the cell, Bull. Math. Biol. 56 (1994) 587.

[15] A. Suciu, G. Civelekoglu, Y. Tardy, J.J. Meister, Model for the alignment of actin filaments in endothelial cells subjected to fluid shear stress, Bull. Math. Biol. 59 (1997) 1029.

[16] G.B. Ermentrout, L. Edelstein-Keshet, Models for contact-mediated pattern formation: cells that form parallel arrays, J. Math. Biol. 29 (1990) 33.

[17] A. Mogilner, L. Edelstein-Keshet, Spatio-angular order in populations of self-aligning objects: formation of oriented patches, Physica D 89 (1996) 346.

[18] A. Stevens, Trail following and aggregation of myxobacteria, J. Biol. Sys. 3 (1995) 1059.

[19] A. Deutsch, Towards analysing complex swarming patterns in biological systems with the help of lattice-gas cellular automata, J. Biol. Sys. 3 (1995) 947.

[20] J. Cook, Waves of alignment in populations of interacting oriented individuals, Forma 10 (1995) 171.

[21] D. Grübaum. Advection-diffusion equations for generalised tactic searching behaviors. J. Math. Biol. (in press).

[22] L. Olsen, P.K. Maini, J.A. Sherratt, B. Marchant. Simple modelling of extracellular matrix alignment in dermal wound healing. i. Cell flux induced alignment. J. Theor. Med. 1 (1998) 175 .

[23] J.C. Dallon, J.A. Sherratt. A mathematical model for fibroblast and collagen orientation. Bull. Math. Biol. (in press).

[24] G.J. Pettet, H.M. Byrne, D.L.S. McElwain, J. Norbury. A model of wound-healing angiogenesis in soft tissue. Math. Biosci. (in press).

[25] M.A.J. Chaplain, H.M. Byrne, Mathematical modelling of wound healing and tumour growth -2 sides of the same coin, Wounds: A Compendium of Clinical Research and Practice 8 (1996) 42.

[26] L. Olsen, P.K. Maini, J.A. Sherratt, F. Arnold, A Mathematical model for the capillary endothelial cell-extracellular matrix interactions in wound-healing angiogenesis, IMA J. Math. Appl. Med. Biol. 14 (1997) 261.

[27] R.T. Tranquillo, J.D. Murray, Continuum model of fibroblast-driven wound contraction: Inflammation-mediation, J. Theor. Biol. 158 (1992) 135.

[28] L. Olsen, J.A. Sherratt, P.K. Maini, A Mechanochemical model for adult dermal wound contraction and the permanence of the contracted tissue displacement profile, J. Theor. Biol. 177 (1995) 113.

[29] J.D. Murray, J. Cook, R. Tyson, S.R. Lubkin, Spatial pattern formation in biology: I. Dermal wound healing II. bacterial patterns, J. Franklin Inst. 335 (1998) 303.

[30] P.D. Dale, J.A. Sherratt, P.K. Maini, A mathematical model for collagen fibre formation during foetal and adult dermal wound healing, Proc. Roy. Soc. Lond. B 263 (1996) 653.

[31] D. Stopak, A.K. Harris, Connective tissue morphogenesis by fibroblast traction I: Tissue culture observations, Dev. Biol. 90 (1982) 383.

[32] J. B. McCarthy, D. F. Sas, L. T. Furcht, Mechanisms of parenchymal cell migration into wounds, in: R.A.F. Clark, P.M. Henson, (Eds.), The Molecular and Cellular Biology of Wound Repair Ch. 13, Plenum, New York, 1988, p. 281.

[33] R.L. Trelstad, K. Hayashi, Tendon collagenfibrillogenesis: Intracellular subassemblies and cell surface changesassociated with fibril growth, Dev. Biol. 71 (1979) 228.

[34] S. Guido, R.T. Tranquillo, A methodology for the systematic and quantitative study of contact guidance in oriented collagen ges, J. Cell Sci. 105 (1993) 317.

[35] A. Mogilner, L. Edelstein-Keshet, Selecting a common direction. How orientational ordercan arise from simple contact responses between interacting cells, J. Math. Biol. 33 (1995) 619. 
[36] E. Bell, B. Ivarsson, C. Merrill, Production of a tissue-like structure by contraction ofcollagen lattices by human fibroblasts of different proliferative potential in vitro, Proc. Natl. Acad. Sci. USA 76 (1979) 1274.

[37] T.J. Ryan, Biochemical consequences of mechanical forces generated bydistention and distortion, J. Am. Acad. Dermatol. 21 (1989) 115.

[38] R.B. Vernon, E.H. Sage, Between molecules and morphology. Extracellular matrix and creation of vascular form, Am. J. Pathol. 147 (1995) 873.

[39] J.A. McDonald, Fibronectin: A primitive matrix. In: R.A.F. Clark, P.M. Henson, (Eds.), The Molecular and Cellular Biology of Wound Repair, Ch. 18, Plenum, New York, 1988, 405.

[40] M.P. Welch, G.F. Odland, R.A.F. Clark, Temporal relationships of F-actin bundle formation collagenand fibronectin matrix assembly and fibronectin receptor expression to woundcontraction, J. Cell Biol. 110 (1990) 133.

[41] R.A.F. Clark, Biology of dermal wound repair, Dermatol. Clin. 11 (1993) 647.

[42] P. Gillery, F.-X. Maquart, J.-P. Borel, Fibronectin dependence of the contraction of collagen latticesby human skin fibroblasts, Exp. Cell Res. 167 (1986) 29.

[43] I.I. Singer, D.W. Kawka, D.M. Kazais, R.A.F. Clark, In vivo co-distribution of fibronectin and actin fibersin granulation tissue: Immunofluorescence and electron microscope studies ofthe fibronexus at the myofibroblast surface, J. Cell Biol. 98 (1984) 2091.

[44] D.E. Birk, R.L. Trelstad, Extracellular compartments intendon morphogenesis: Collagen fibril bundle and macroaggregateformation, J. Cell Biol. 103 (1986) 231.

[45] J.C. Dallon, J.A. Sherratt, P.K. Maini, Collagen alignment in discrete mathematical models of matrix orientation and tissue regeneration, submitted.

[46] J.C. Dallon, Numerical aspects of discrete and continuum hybrid models in cell biology, submitted.

[47] A. Ralston, P. Rabinowitz, A first course in numerical analysis, McGraw-Hill New York, 1978. 